\title{
$\left[{ }^{11} \mathrm{C}\right]$ PBB3 - a new PET ligand that identifies tau pathology in the brains of patients with AD
}

$\mathrm{R}$ esearchers from Japan and the USA have developed a new PET radioligand that can be used to detect tau deposits in the brains of living individuals. This approach could provide important insights into an aspect of the Alzheimer disease $(\mathrm{AD})$ process that has not previously been amenable to in vivo analysis.

"Recent failures in clinical trials of anti-amyloid- $\beta$ (A $\beta)$ treatments, as exemplified by $A \beta$ immunotherapies, have led to a view that these therapies do not suppress AD progression unless the drugs also diminish tau pathologies," explains Makoto Higuchi, who led the study. "On this basis, a number of research groups started focusing on the development of PET imaging agents for tau lesions.”

Higuchi and his colleagues faced two major challenges in the development of a PET radioligand for tau imaging. First, the candidate ligands were designed to target $\beta$-pleated sheets, a protein structural motif that is shared by tau and $A \beta$, thereby raising the possibility of cross-reaction. Second, the ligand needed to be able to cross the blood-brain barrier (BBB).

The researchers developed a range of fluorescent compounds known as PBBs. These compounds were screened for their capacity to detect tau in brain tissue from patients with AD, and from PS19 mice, which overexpress a mutant form of human tau. "Among these compounds, $\mathrm{PBB} 3$ was suitable as a PET probe, since it showed reasonable biostability and selectivity for tau," says Higuchi.

The team initially tested PBB3 in PS19 mice by means of in vivo two-photon laser microscopy. After intravenous injection, the compound was found to rapidly enter the CNS, where it bound to intracellular tau aggregates. A subsequent PET imaging study using an ${ }^{11}$ C-radiolabelled form of PBB3 in PS19 mice confirmed that the ligand could detect tau in the living brain.

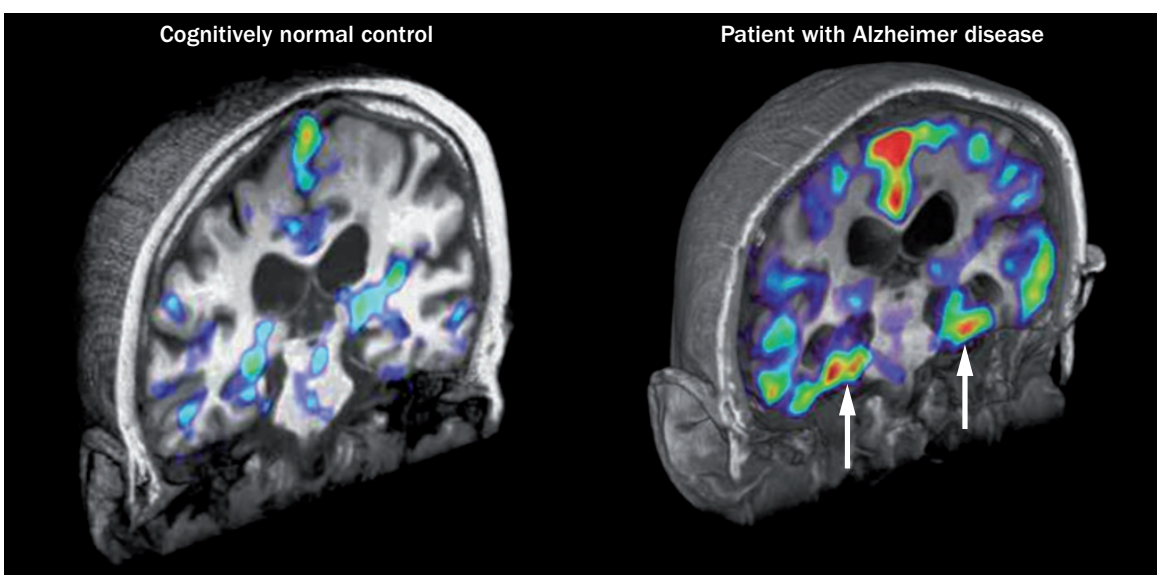

The PET imaging ligand $\left[{ }^{11} \mathrm{C}\right] \mathrm{PPB} 3$ reveals tau accumulation in the hippocampal formation (arrows) in a patient with Alzheimer disease. Image courtesy of M. Higuchi.

Higuchi and colleagues went on to compare the results of PET imaging with $\left[{ }^{11} \mathrm{C}\right] \mathrm{PBB} 3$ and the $\mathrm{A} \beta$-imaging compound $\left[{ }^{11} \mathrm{C}\right] \mathrm{PIB}$ in three patients with probable $\mathrm{AD}$ and three age-matched cognitively normal controls. In the patients with $\mathrm{AD}$, the $\left[{ }^{[1} \mathrm{C}\right] \mathrm{PBB} 3$-PET signal was especially strong in the hippocampal formation, whereas retention of $\left[{ }^{11} \mathrm{C}\right] \mathrm{PIB}$ was minimal in this region. The findings were consistent with previous observations on the distribution of tau and $A \beta$ pathology in the brains of patients with $\mathrm{AD}$.

"[ $\left.{ }^{11} \mathrm{C}\right] \mathrm{PBB} 3-\mathrm{PET}$ demonstrated spreading of brain tau pathology in transition from normal ageing to moderate $\mathrm{AD}$, suggesting the usefulness of tau PET imaging as an objective index of disease progression," reports Higuchi. "This was in sharp contrast to the intensity and distribution of $\left[{ }^{11} \mathrm{C}\right]$ PIB-PET signals, which barely changed after the clinical onset of $\mathrm{AD}$, and is in agreement with the notion that tau lesions are more tightly associated with neuronal loss than are $A \beta$ plaques."

$\left[{ }^{11} \mathrm{C}\right] \mathrm{PBB} 3$ is not the only tau radioligand currently in development-Hartmuth Kolb and co-workers recently reported promising results from clinical studies with the ${ }^{18}$ F-labelled PET ligands [F18]-T807 and [F18]-T808. The comparatively long radioactive half-life of ${ }^{18} \mathrm{~F}$ facilitates the transport of these compounds between radiosynthesis and imaging facilities, but $\left[{ }^{11} \mathrm{C}\right] \mathrm{PBB} 3$ seems to have other practical advantages, including an affinity for a variety of tau lesion types, and reduced exposure of patients to radioactivity.

Higuchi's team is now planning a multicentre study to validate $\left[{ }^{11} \mathrm{C}\right] \mathrm{PBB} 3$ as a tau radioligand in a range of tauopathies. They also anticipate that the ligand could be used to monitor the efficacy of anti-tau drugs. "These interventional approaches, along with in vivo monitoring, should eventually provide important evidence for the mechanistic contribution of tau accumulation to neuronal dysfunction and death, and emergence of symptoms, in diverse tau-positive neurodegenerative disorders," concludes Higuchi.

Heather Wood 